\title{
Research and realization of an improved auto-correlation method for frequency measuring
}

\author{
Wang Liu, Hai-Qi Zhang and Ming-Hao Zhong \\ JiuQuan Satellite Launch Center, Gansu province, China \\ E-mail: paper_woodyduke@sina.com
}

\begin{abstract}
Frequency measuring is an important part of modern radar systems. Based on the traditional principle of auto-correlation algorithm, we put forward an improved method of frequency measurement, simulating and analyzing the implementation process with MATLAB, finally testing on FPGA platforms. The results show the correctness and feasibility of the method, highlighting the theoretical and practical guiding significance to the design of a fast frequency measurement system for digital receivers.
\end{abstract}

Keywords: Frequency Measurement; Synthesizers; Auto-Correlation; FPGA.

\section{Introduction}

Digital frequency measurement system is an important part in signal processing, frequency precision extraction has a profound impact on the implement of technology during the procedure, such as target stealth and signal interception and some other technology in digital signal intelligence. At present, radar absorbing materials has been widely used in radar stealth systems, it is a type of radars with frequency selective surface (Frequency Selective Surface---FSS), which distributes the signal energy out of the frequency range in enemy' radar receivers while under the radiation, and indirectly control the reflecting rate consequently, lead to the unsuccessful detection of appropriate echo signals in enemy's radar systems. During the detection of radar signals with a smart waveabsorbing structure, the scope of external voltage with absorbing materials' is based on the radar pulse signal frequency, which results from the control module in digital radar measuring system. As we can conclude that the frequency measuring technology of digital signals is a key part in electronic reconnaissance systems, and in addition, the signal frequency measuring technology plays a very important role in reconnaissance for enemy signals and electronic intelligence also.

Based on the traditional principles and MATLAB Simulation, we propose a method for variable frequency synthesizer with auto-correlation frequency 
measuring technology, and fully design a fast signal frequency measurement module for radar signal, and use the Verilog Hardware language for hardware implementation on FPGA platform.

\section{Overview of Auto-correlation Frequency Measuring Method}

Typically, methods for radar signals' detection can be divided into two large categories, which are frequency-domain and time-domain detection [1] [2]. In the detection of frequency-domain category, wavelet analysis and Fourier transform approaches are widely used, which mainly based on theory that radar signals energy distribute in a smaller range of frequencies in frequency domain. Detect the frequency of radar signal where energy focused after input signal transform to the frequency-domain, then we get the initial and end time of the input signals after transform the frequency to time-domain in the end. As it can be seen, much more precise results can be obtained with frequency-domain detecting process, but frequency-domain detecting process needs double domain transformations, which brings high-computational work to the hardware unit and is not conducive for fast real-time detection in radar systems.

Conversely, time-domain detecting methods contain the accumulator and auto-correlation processes only, interference signals in the input signal is white Gaussian noises, which is irrelevant, not to speak of the frequency characteristic, while the useful radar signals have independent frequency characteristics. Under the superposition with radar pulse itself, signal amplitude and energy will increase persistently and noise signals still to be white Gaussian noises itself no matter how many times of superposition. With the auto-correlation operation, useful pulse energy can be accumulated continuously, while noises vanish in the meantime, signal-to-noise ratio get improved consequently that benefit to the extracting of radar signals.

Actually, with reducing of calculation, improving of signal-to-noise ratio and increasing real-time performance, auto-correlation methods in time-domain can be used to detecting useful radar signals with a very low SNR (signal-tonoise ratio). The implement of frequency measuring can be simplified with using cumulative structure without the transformation between time-domain and frequency-domain, easier to be realized on a limited hardware platform at the same time.

During frequency extracting process of the useful radar signals, discrete complex signal sequences with finite signal energy, $r_{x y}(l)$ can be defined as cross-correlation sequence, thus 


$$
r_{x y}(l)=\sum_{n=-\infty}^{\infty} x(n) y^{*}(n-l), l=0, \pm 1, \ldots
$$

or

$$
r_{x y}(l)=\sum_{n=-\infty}^{\infty} x(n+l) y^{*}(n), l=0, \pm 1, \ldots
$$

In the equations, $l$ is a delay parameter in convolution, $y^{*}(n)$ is the conjugate result of $y(n)$, if $x(n)=y(n)$, the auto-correlation sequence $r_{x x}(l)$ can be obtained based on cross-correlation of $x(n)$ :

$$
r_{x x}(l)=\sum_{n=-\infty}^{\infty} x(n) x^{*}(n-l), l=0, \pm 1, \ldots
$$

and

$$
r_{x x}(l)=\sum_{n=-\infty}^{\infty} x(n+l) y^{*}(n), l=0, \pm 1, \ldots
$$

As a matter of fact, signal $x(n)$ is a causal sequence with finite length in the processing, and when the length of $x(n)$ is set to $\mathrm{N}$, when $n<0$ and $n \geq N$, auto-correlation sequences $r_{x x}(l)$ can be expressed as:

$$
r_{x x}(l)=\sum_{n=i}^{N-|k|-1} x(n+l) x^{*}(n), l=0, \pm 1, \ldots
$$

when $l \geq 0, i=0 、 k=l$, and $l<0, i=l 、 k=0$, the down-converted signal in the radar receiver $x(n)$ can be defined as :

$$
x(n)=I(n)+j Q(n), 0 \leq n \leq N-1
$$

$I(n)$ and $Q(n)$ is in-phase and quadrature components of the signal, which can be expressed as:

$$
\begin{aligned}
& I(n)=A(n) \cos \left(2 \pi f_{n} / f_{s}+\varphi_{n}\right) \\
& Q(n)=A(n) \sin \left(2 \pi f_{n} / f_{s}+\varphi_{n}\right)
\end{aligned}
$$

$f_{m}$ is signal frequency and $f_{s}$ is the sampling frequency in the equations. After auto-correlation processing, results can be: 


$$
R(n)=A(n) A(n+m)\left\{\cos \left(2 \pi f_{m} / f_{s}\right)-j \sin \left(2 \pi f_{m} / f_{s}\right)\right\}
$$

the phase turns out to be:

$$
\psi_{n}(n, m)=-2 \pi f_{m} / f_{s}
$$

Depends on the DDS technology, the input radar signal instantaneous frequency can be obtained from the phase [3] [4] .

However, since auto-correlation process needs to be associated with ergodic calculation with the sampling data. Large amounts of computation comes in the hardware processor, in addition to a low frequency accuracy gotten by the use of algorithm in auto-correlation signal processing at the same time, leading to severely limited measuring accuracy for aircraft's speed and distance in radar systems. Thus we propose an improved processing method in the following text, which can be used to improve the resolution of frequency. With the improved implement method, auto-correlation results can be extracted instantaneously from an interception of a signal $\mathrm{x}(\mathrm{n})$. With the Phase information putting into the DDS module, frequency extracting process is completed in real-time ${ }^{[5]}$, not merely increase the speed of frequency detection and measuring, but also improve the radar distance and speed measurement accuracy.

\section{Design of Frequency Measuring}

During the design of frequency measurement module, radar pulse lasts about 150ns (pulse in the single-frequency sine wave), sampling frequencies of the system requirements is $1.8 \mathrm{G}$, and then $270(1.8 \mathrm{G} * 150 \mathrm{~ns}=270)$ valid data can be drawn in the sampling process. For facilitating the measuring process, we choose 256 data in the auto-correlation operations. According to the second Nyquist sampling law, under the $1.8 \mathrm{GHz}$ sampling clock, not fuzzy frequency interval exist in $0.9 \mathrm{GHz}$ to $1.8 \mathrm{GHz}$ since the frequency of the signal lies in the range of $1.0 \mathrm{GHz}$ to $1.5 \mathrm{GHz}$.

First of all, square waves can be obtained by the comparison of sampled sinusoidal signals and a fixed threshold value, we set the square wave comparative results to 1 when sinusoidal signal amplitude is greater, inversely to 0 when the amplitude is lower than that of the threshold value. Thus the squarewave spectrum contains the original signal frequency information and mixes a lot of harmonic components. With delaying of 1,2,4,9 system clock for square wave signal respectively and XOR operating with the original square, we count the numbers of 1 in the XOR results, get a graph of auto-correlation values related to the frequency of original signals in the following Figure 1. 


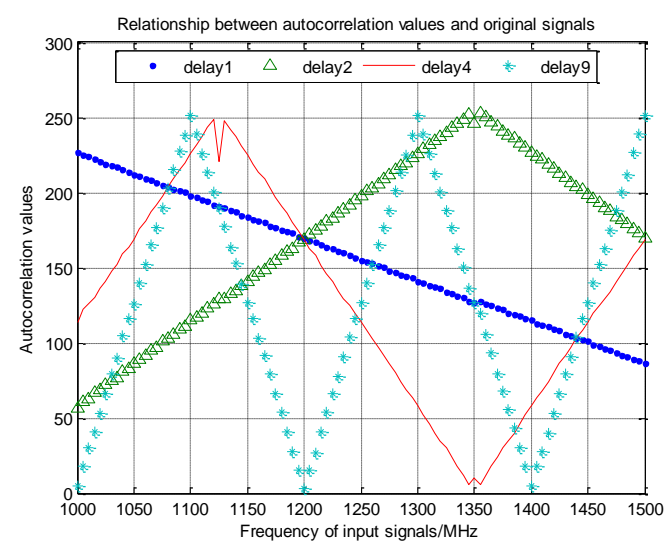

Fig. 1 Signal frequency and related auto-correlation values

With the of delay 1 auto-correlation values, the frequency accuracy can be obtained up to $3.53 \mathrm{MHz}(900 \mathrm{MHz} / 255=3.53 \mathrm{MHz})$. Combined with the noises in the signal, a rough estimate for the frequency can only be made with a delay of 1 system clock, and can't able to provide a desired frequency precision and resolution, and make a limitation for the accuracy of velocity and distance in the radar systems consequently.

As a matter of fact, the auto-correlation can be changed on account of a very small change in frequency, thus we can increase the number of delays what will lead to an increasing precision and resolution in the measuring.

From the relationship between auto-correlation and frequency of input signal values in Fig. 1, we can conclude that with the frequency of input signal range from the $1000 \mathrm{MHz}$ to $1500 \mathrm{MHz}$, auto-correlation values for one system clock delays is linear associated with the signal frequency, while there has been a turning point in the auto-correlation values with two system clock delays, the auto-correlation values' variation range with 4 delays is about 2 times contrast to the Delay2, and non-linearity with 9 system clocks delays becomes more prominent .

As we can see in the Fig.1, only the curve of Delay1 is corresponding to the signal frequency one by one and without any fuzz, while the other three curves of more delays lies in fuzzy on specific frequency. The theoretically frequency accuracy of Delay4 turns out to be $0.88 \mathrm{MHz}(900 \mathrm{MHz} /(256 * 4)=0.88 \mathrm{MHz})$, meeting with the requirement for frequency measurement system basically. But we still need solutions to remove the blur because fuzz appears in line of Delay4. To guarantee the calculating speed and improve accuracy, we can accomplish the defuzzification according to the comprehensive results of different delays. 
Based on the auto-correlation process described, we propose an improved auto-correlation measurement method. Firstly, a rough initial value of frequency can be calculated based on the relationship between the auto-correlation values and frequency of input signals in line of Delay1, which determines the existence range of the measured signal frequency. And then extract an accurate frequency with corresponding relation put in line of Delay 4, by guaranteeing the effectiveness of frequency measuring accuracy and improving the speed of the measurement module at the same time. A poor linearity lies in Delay4 on specified frequency show in the Figure 1.

As it shown in Figure 1, gross errors appear when we calculate the frequency with the line of Delay4 in poor linearity range of $1120 \mathrm{MHz}$ and $1350 \mathrm{MHz}$. As a solution, we consider about this interval of the line with two delays for calculating, but in the line of Delay2, two disadvantages exist: one is that the frequency accuracy is lower than that of Delay4 results, and another is that the linearity of line Delay2 is also not ideal in $1350 \mathrm{MHz}$, hence we need to consider further options for frequency measuring.

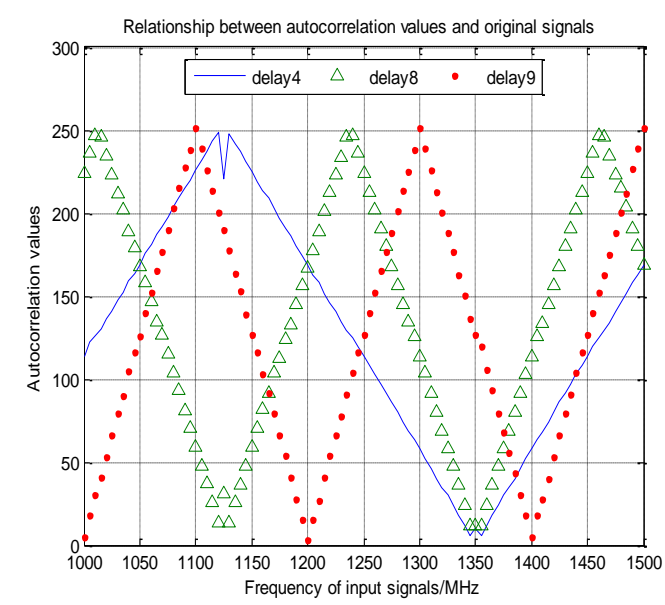

Fig. 2 Auto-correlation values of Delay4 and Delay8

Auto-correlation values related to the input signal show in Figure 2. Inflection points exist in specified frequency in line of Delay4 and Delay8, because of delayed results are powers of integer 2 , so it can be determined that further more accuracy relies on the auto-correlation processing excluding the delay with power of integer 2 . 
It can be seen in the Figure 2 that Delay9 has a greater variation than that of the Delay 4, nonlinear intervals in Delay4 is strictly linear in line of Delay9, so we can take the values in Delay 9 to solve nonlinear problems in the Delay4.

Thus we can choose the auto-correlation values with Delay1 and Delay4 and Delay9 to solve the ambiguity in frequency measurement. We find the range of frequency with Delay1, measuring the frequency accurately with the relationship shown in Delay4, and choose the corresponding relation of Delay9 to exclude the fuzzy intervals of Delay4. Fitting results of frequency with MATLAB simulation show in the following figure 3.

Simulation base on the lower order of fitting function in MATLAB, and the frequency measuring results correspond to relationship between auto-correlation values and input radar signals shown in Figure 3.
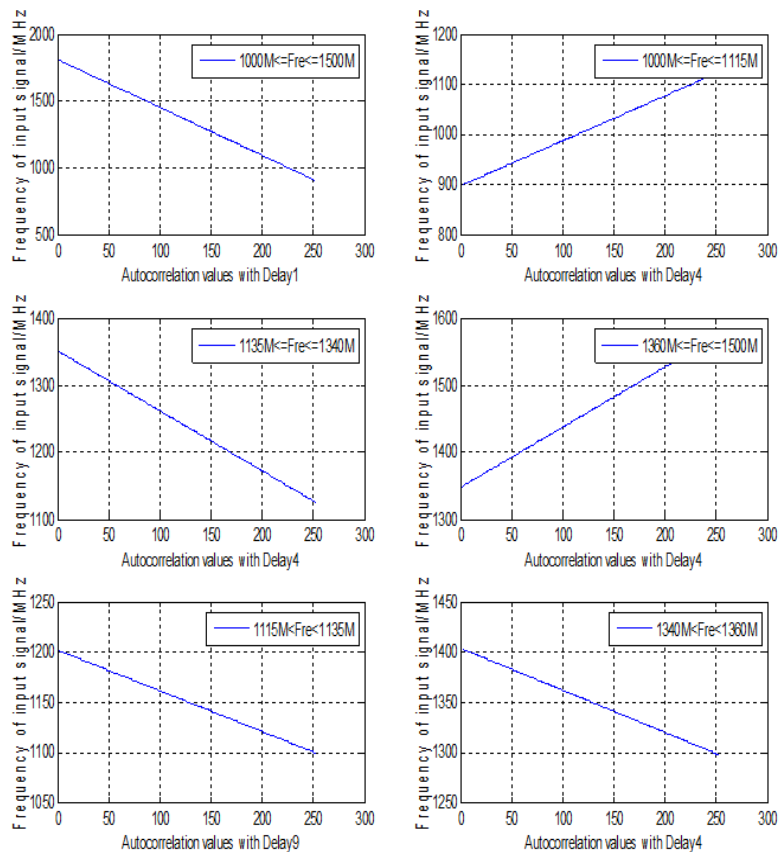

Fig. 3 Frequency measurement results of MATLAB simulation

Range of frequency can be confirmed quickly with curve Delay1, and accurately measuring the frequency with the relationship shown in curve Delay4, the fuzzy lies in Delay4 can be excluded while choosing the corresponding relation of Delay9. Lastly, we utilize the DDS frequency synthesizer with frequency generated by the proposed technology. At this point, the measurement 
of signal frequency and frequency synthesis signal generation is accomplished completed.

\section{Implementation and Testing on FPGA Platform}

During the hardware realization of radar receiver system platform, 1500M input signal comes from a signal source. Based on the theoretical simulation with MATLAB, input signals are sampled by the chip of ADC083000 with a $1.8 \mathrm{GHz}$ sampling clock, thus the rate of output DDR data at each ADC quad port is $225 \mathrm{MHz}$. After the buffer processing, system clocks decrease to $112.5 \mathrm{MHz}$ on FPGA platform and 16 points are sampled at each system clock. Therefore, the input radar signals get sampled with $256(16 * 16=256)$ data within 16 system clocks [6] [7].

Secondly, square wave signals generated by the comparing of sampling value and the fixed threshold. As we know, chip of ADC083000 output is represented by a 8-bit unsigned number, thus we set the threshold value as ox 80 , and square wave signal can be formed while comparing the sampled signal data with threshold values.

Thirdly, we set the square wave signals with one, four and nine system clocks, which means that moving back the data according to the sampling time and fill with zeros in the front, with the XOR operation between the delayed data and original square wave.

Lastly, accumulate the number of XOR operation with one, four and nine system clocks' delay, which represent the strictly linear relationship between input signals and the auto-correlation values. Results of the hardware implementation on FPGA platform show in Figure 4. 


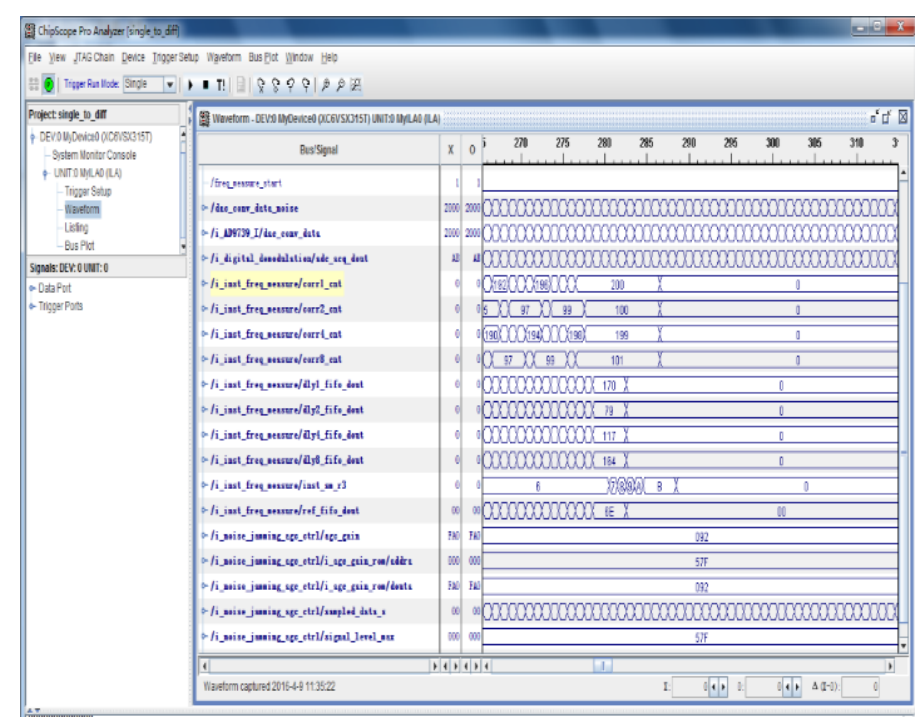

Fig. 4 Hardware processing of the results

It shows that corr1-corr8 is corresponding to different delays process in the software associated with the auto-correlation values in the figure 4 . In the hardware implementation, we can get the accurate signal frequency quickly by querying the frequency data tables in the buffer cache. And according to the querying results, frequency synchronized signal can be produced by the calculated frequency with DDS (Direct Digital Synthesizer). In fact, AD9739 chip can work in normal mode and mixing mode, in this project we set the mode in the mixing mode, eventually the frequency measurement can be implemented on hardware platform, and sent the frequency synchronized signal to the radar receiver demodulation unit, fulfill the demodulation processing rapidly.

\section{Summary}

This paper introduces a kind of improved auto-correlation processing for rapid implementation of frequency measurement. Through the auto-correlated operations and defuzzificating processing, frequency can be extracted accurately. Signal generated directly by DDS guarantee the accuracy of the frequency, and improving the digital receiver frequency measuring speed further more. This paper carries out theoretical analysis of the frequency measurement method, and makes use of MATLAB for the simulation and analysis of implementation process, results which coming from hardware implementation on FPGA 
platform prove the correctness of the method, highlight the theoretical and practical significance for frequency measurement of digital receiver.

\section{References}

1. Frequency measuring system based on FPGA for radar signal [D]. HuaZhong University of science and a master's thesis, 2008.

2. Liu Xinqun, Auto-correlation algorithm and its application in digital instantaneous frequency measurement receivers [J], electronic counter measures, 2008 (2) 22-25.

3. Dong Hui, Measurement of instantaneous frequency in digital reconnaissance techniques[J], electronic countermeasures , 2005 (5): 7-10.

4. Delpart N.etc, A symptomatic wavelet and Gabor Analysis: Extraction of instantaneous frequencies [J]. IEEE Transactions, 1992, 38(3):644- 664.

5. Peter Hessler, New digital wide band Receiver [D]. University doctoral thesis, 2004.

6. Young calves, Principle and application of software radio [M]. Beijing: Publishing House of electronics industry,2001.

7. Lixiang,Ren Shufen Ma, Fanghui Li, Theory and Application of TMS320C6000 series DSP [M]. Beijing: Publishing House of electronics industry, 2000.285-289. 